\title{
Multi-hop Fast Conflict Resolution Algorithm for Ad Hoc Networks
}

\author{
Shengwei Wang ${ }^{1}$, Jun $\mathrm{Liu}^{2, *}$, Wei $\mathrm{Cai}^{2}$, Minghao $\mathrm{Yin}^{2}$, Lingyun $\mathrm{Zhou}^{2}$, and $\mathrm{Hui} \mathrm{Hao}^{3}$ \\ ${ }^{1}$ Power Emergency Center, Sichuan Electric Power Corporation, Chengdu, China \\ ${ }^{2}$ National Key Laboratory of Science and Technology on Communications, University of Electronic \\ Science and Technology of China, Chengdu, China \\ ${ }^{3}$ Information Technology Center, Tsinghua University, Peking, China
}

\begin{abstract}
Media access control is an important technology in ad hoc networks. Based on the multi-hop Pseudo Bayesian (PB) algorithm and the single-hop Fast Conflict Resolution algorithm (FCR), in this paper, we propose a Multi-hop Fast Conflict Resolution (MFCR) algorithm for ad hoc networks. We make the detailed introduction of the theoretical model, the pseudo Bayesian analysis and the algorithm deign. MFCR employs a competition probability forecasting method, which is similar to the backoff counter used in FCR, combined with the handshake process used in PB to solve the utilization and the fairness problems of the time slot allocation. Simulations show that the MFCR has evident improvement in the network performance.
\end{abstract}

\section{Introduction}

The media access control is an important technology in Ad-Hoc networks [1]. Because of the limitation of frequency, the wireless channel resource is relatively scarce. Meanwhile, the channel fading, the interference and the transmission collision will deteriorate the transmission capacity [2][3]. Hence, the channel resource allocation will play an important role. The channel of Ad-Hoc networks is a multi-hop broadcast shared channel, only the nodes are far away from each other can reuse the same frequency at the same time [4].

In Time Division Multiple Access (TDMA) Media Access Control (MAC) protocol [5][6][7], the wireless channel is divided into periodic frames in time dimension, and each frame consists of some time slots. The goal of channel resource allocation is making nodes rationally utilize limited slot resource according to the network situation.

The static TDMA protocol can't adjust the slot allocation scheme to the network situation, which will cause the waste of the channel resource. In contrast, the dynamic TDMA protocol can adjust the slot allocation flexibly to adapt to the network situation [8], so it can effectively use the slot resource.

The Five-Phase Reservation Protocol (FPRP) is a typical dynamic TDMA protocol based on competition [9]. The nodes in the network obtain their own delivery slot through competition mechanism, so the slot allocation of each time frame is different.

*Corresponding author: liu_jun@uestc.edu.cn 
Due to the distributed characteristic, the FPRP protocol can be executed independently by each node. That is to say, the nodes more than two hops can simultaneously use the same slot. For each node, the slot reservation process can be regarded as a local reservation procedure, which only involves the slot reservation within two hops. Through the local reservation process, it makes the network have a good scalability.

In FPRP protocol, the single wireless channel is logically divided into the reservation channel and the information channel. To TDMA frame, it is the reservation frame and the information frame. Each reservation frame and information frame has the same number of time slots. In the reservation phase, the node reserves time slot by a five phase handshake mechanism. In this way, FPRP can realize a conflict-free slot allocation for packet delivery. The reservation frame is followed by a number of information frames, the nodes in the network can transmit data in the information slot until the next reservation frame.

\section{System model}

We use graph $G(V, E)$ to represent the network topology, where $V$ is the node set and $E$ is the link set in the network. If there is a link between node $i$ and node $j$, we use an undirected edge $e_{\mathrm{i}, \mathrm{j}}, e_{\mathrm{i}, \mathrm{j}} \in \mathrm{E}$, to represent the one-hop neighboring relationship. If there is no direct link between node $i$ and node $j$, but they have a common neighbor $k$, we define node $i$ and node $j$ as two-hop neighbor. We use matrix $\mathbf{C}(\mathrm{G})=\left[c_{i j}\right]_{N \mathrm{x}_{N}}$ and $\mathbf{D}(\mathrm{G})=\left[d_{i j}\right]_{N \mathrm{x}_{N}}$ to represent the one-hop connection relationship and the two-hop connection relationship respectively 10. The $c_{i j}$ and $d_{i j}$ are defined as

$$
\begin{gathered}
c_{i j}= \begin{cases}1, & e_{i j} \in E, i \neq j \\
0, & \text { else }\end{cases} \\
d_{i j}= \begin{cases}1, & e_{i k}, e_{j k} \in E, e_{i j} \notin E, i \neq j \\
0, & \text { else }\end{cases}
\end{gathered}
$$

Owing to the nodes in a half-duplex wireless network cannot receive multiple packets simultaneous and cannot transmit and receive at the same time 11, the time slot of each node must be different from the one-hop neighbors and the two-hop neighbors to avoid transmission collision.

The main goal of the slot assignment algorithm is to maximize the channel utilization with the minimum number of time frames. Consider a time frame has $M$ slots and each node's slot number is at least one. We use a $M \times N$ matrix $\mathbf{T}=\left[t_{m i}\right]_{M \times N}$ to represent the slot allocation scheme in network. If time slot $m$ belongs to node $i$, we set $t_{m i}=1$, otherwise $t_{m i}=0$, and define the slot utilization of node $i$ as

$$
\rho_{i}=\frac{\sum_{m=1}^{M} t_{m i}}{M}
$$

The slot utilization of the whole network can be expressed as

$$
\rho=\frac{1}{N} \sum_{i=1}^{N} \rho_{i}=\frac{1}{M N} \sum_{m=1}^{M} \sum_{i=1}^{N} t_{m i}
$$

As a result, the slot allocation can be summarized as the following mathematical model 


$$
\begin{gathered}
\min M \text { and max } \rho \\
\text { s.t. } C 1: \sum_{m=1}^{M} t_{m i} \geq 1 \quad(i \in N) \\
C 2: c_{i j}+t_{m i}+t_{m j} \leq 2(i, j \in N, i \neq j, m \in M) \\
C 3: c_{i k} t_{m i}+c_{j k} t_{m j} \leq 1 \quad(i, j, k \in N, i \neq j \neq k, m \in M)
\end{gathered}
$$

where $C 1$ denotes that each node takes at least one slot, $C 2$ represents two one-hop neighbors can't be allocated with the same slots, $C 3$ stands for that two-hop neighbors can't use the same slots.

\section{Pseudo baysian algorthm}

In this paper, we use the Pseudo Bayesian algorithm 12 to calculate the probability of nodes participating in the slot reservation. Through the five phase handshake reservation mechanism in FPRP, the node in the network can sense the current slot reservation status, such as success, idle, and conflict. Because the reservation process is a local process within two-hop, the node needs to maintain two parameters: one is the number of competition nodes within its two-hop; the other is the number of nodes want to reserve the slot, but can't compete for the current slot due to the slot being reserved by others. When a node is informed that the current slot is allocated to one of its neighbors, it stops the competition for the current slot, but can still participate in the competition for the next slot. The status of idle, conflict, and success will affect the number of competition neighbors within two-hop, so the nodes can participate in the remaining reservation process with a modified competition probability.

According to Pseudo Bayesian algorithm, the competition probability of each node is related to the number of competing nodes within two-hop. That is, the number of competitors in two-hop is inversely proportional to the competition probability. Consider two situations in the network, the one is in the dense area (such as the network center area), the other is in the sparse area (such as the edge of the network). Because there are more neighbors in the dense area, more reservation activities will take place, and there will be more conflicts. As it is hard to find the idle state in the reservation process, the possibility of decreasing the competition probability is high. On the other hand, because there are fewer neighbors in the sparse area, there will be fewer conflicts, and it is easier to sense the idle state in the reservation process. According to the Pseudo Bayesian algorithm, once a node finds an idle state in the current sot reservation process, the competition probability will be increased. So the possibility of increasing the competition probability in the sparse area is higher than that in the dense area. Thus, the difference of node density in two-hop will result in unfair slot allocation.

\section{Multi-hop fast conflict resolution algorithm}

In order to solve the problem in multi-hop pseudo Bayesian algorithm, we propose a Multihop Fast Collision Resolution (MFCR) based on single-hop Fast Collision Resolution (FCR) [13]. In FCR, when the node senses the idle slot, the value of back off counter decreases by one. When a node checks continuously idle slots, once the slot been idle again, the value of back off counter decreases by half. On the other hand, when a node senses the collision, it doubles the value of contention window (CW). Set a random number between $0-\mathrm{CW}$ as the value of back off counter. In this way, when collision emerges, the node defers its channel access to decrease the collision possibility. 
MFCR applies the Fast Conflict Resolution in the multi-hop ad hoc networks. In MFCR, $n_{c}$ is used to predict the number of neighbors within two-hop, the role of $n_{c}$ is similar to the back off counter used in FCR. The larger $n_{c}$ is, the smaller the competition probability of a slot achieves. Considering the fairness of slot allocation, we limit $n_{c}$ between $n_{\min }$ and $n_{\max }$. Through the five-phase handshake process taken in FPRP protocol, each node can sense the reservation status of the current slot. At the beginning of reservation process, $n_{c}$ is initially set to the minimal value $n_{\min }$. After the reservation process finished in a round, the node updates $n_{c}$ according to the following algorithm.

Algorithm 1 Competition probability adjustment

Input: Reservation status

Output: Competition probability

Note: $x$ denote the number of continuous idle and $k$ is the threshold of continuous idle state, $0<\beta<\alpha<1$. $h$ denote the number of hops to the successful reserving node, and $0<a_{3}<a_{2}<a_{1}<1$. $P$ denote the competition probability.

1. switch ( reservation status)

2. case idle:

3. if $x<k$, then

4. $n_{c}=\max \left(\alpha n_{c}, n_{\min }\right)$

5. else

6. $n_{c}=\max \left(\beta n_{c}, n_{\min }\right)$

7. case conflict:

8. if the node is the reservation initiator

9. $n_{c}=n_{c}+\operatorname{random}\left(0, \min \left(2 n_{c}, n_{\max }\right)-n_{c}\right)$

10. case success:

11. if $h==0, n_{c}=n_{\max }$

12. if $h==1, n_{c}=\max \left(a_{1} n_{c}, n_{\min }\right)$

13. if $h==2, n_{c}=\max \left(a_{2} n_{c}, n_{\text {min }}\right)$

14. if $h \geq 3, n_{c}=\max \left(a_{3} n_{c}, n_{\min }\right)$.

15. end of switch

16. $P=1 / n_{c}$

\section{Then repeat the above steps}

In Idle state, MFCR takes $\alpha$ and $\beta$ as the amplitude factors to multiply by $n_{c}$, the reduction of $\beta$ to $n_{c}$ is greater than that of $\alpha$ to $n_{c}$. When the number of the continuous idle states detected is less than $k$, MFCR uses $\alpha$ to reduce $n_{c}$, and the competition probability of the node increase slowly. If the number of continuous idle state is equal to or bigger than $k$, which means that only a few competition nodes are within two-hop, and the collision probability is low.

When a node encounters a conflict, it will decrease the competition probability rapidly $b$ sharply increase of $n_{c}$. In other words, in MFCR, while the reservation collision occurs within two-hop, the proposed conflict resolution algorithm can decrease the reservation collision rapidly. 
After successfully reserving a time slot, the node updates $n_{c}$ with the maximum value $n_{\max }$ to decrease the competition probability of the next time slot, which guarantees the fairness of slot allocation. If a node gets the information that a one-hop or two-hop neighbor reserves the current slot successfully, the node decreases $n_{c}$ with factor $a_{1}$ and $a_{2}$ to increase the competition probability of the next slot, which speeds up the convergence of slot allocation algorithm. If a node receives a successful reservation message comes from three or more hops away, the node increase its competition probability with factor $a_{3}$ due to the spatial reuse of the time slot 14 .

\section{Performance evaluation}

In this section, the performance of the proposed MFCR is evaluated. We set the network scene in a $1500 \mathrm{~m} \times 1500 \mathrm{~m}$ rectangle area, the communication range is 300 meters. The detailed simulation parameters are listed in Table 1 .

Table 1. Simulation parameters.

\begin{tabular}{|l|l|}
\hline Network range & $1500 \mathrm{~m} \times 1500 \mathrm{~m}$ \\
\hline Communication range & $300 \mathrm{~m}$ \\
\hline Send buffer length & 64 packets \\
\hline Packet size & 1024 bits \\
\hline Packet transmission rate & $1 \mathrm{Mbps}$ \\
\hline$\alpha$ & 0.9 \\
\hline$\beta$ & 0.5 \\
\hline$n_{\min }$ & 2 \\
\hline$n_{\operatorname{maxn}}$ & 24 \\
\hline$a_{1}$ & $1 / 1.1$ \\
\hline$a_{2}$ & $1 / 1.2$ \\
\hline$a_{3}$ & $1 / 1.3$ \\
\hline
\end{tabular}

In addition to the above simulation parameters, we also define the following performance parameters.

Network throughput: The total number of packets sent successfully per second.

Packet delivery rate: The total number of packets received divides by the total packets that the network generates.

Average access delay: The sum of the packet delay of all the received packets divides by the total number of the received packets.

Success rate of competition: The number of nodes that successfully reserve the slot divides by the total number of nodes in the network during a frame time.

Fairness of slot allocation: The variance of slot number allocated to each node in a frame time.

Slot utilization: The total times of successful slot reservation divides by the slot number in a frame time.

\subsection{Average successful competition rate and average time slot utilization rate}

The packet generation rate is set to 20 packet/second/node and the other parameters are the same as table 1. The number of nodes in the network is gradually increased from 20 to 60 . The network topology is randomly generated according to the node number. We simulate the success rate and the average slot utilization of FPRP and MFCR for 20 independent simulations. 


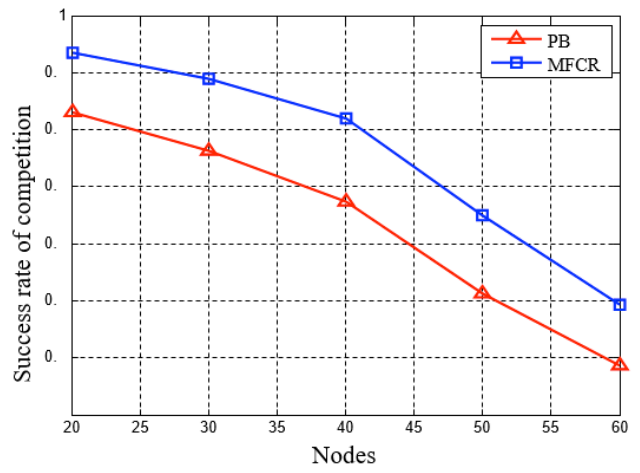

(a) Success rate of competition

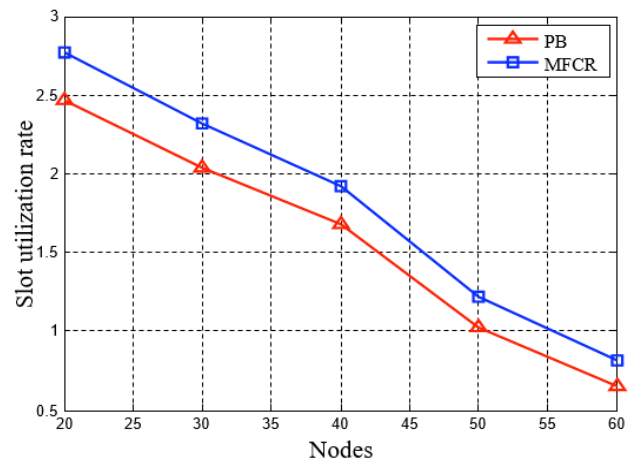

(b) Slot utilization rate

Fig. 1. Comparison of the success rate and the utilization rate.

As shown in Fig. 1(a), the average successful competition rate decreases with the increase of the nodes. The reason is that with the increase of nodes, more conflicts will emerge, thus causing the decline in the success rate of competition. MFCR has a higher success rate than $\mathrm{PB}$, which is because MFCR significantly reduces the competition probability when it encounters conflicts, reducing the potential conflicts in the future.

As shown in Fig. 1(b), the slot utilization rate of MFCR is higher than PB. It reflects that MFCR has a better spatial reuse capability than PB. Moreover, with the increase of the node density, the competitors between two-hop increased, which leads more conflicts. So the slot utilization decreases with the increase of nodes.

\subsection{Fairness of the slot allocation algorithm}

The number of nodes is 25 , packet generation rate is 20 packet/second/node, and the other parameters are the same as table 1 . The network topology is randomly generated according to the node number. We simulate the fairness of slot allocation for MFCR and PB for 20 times.

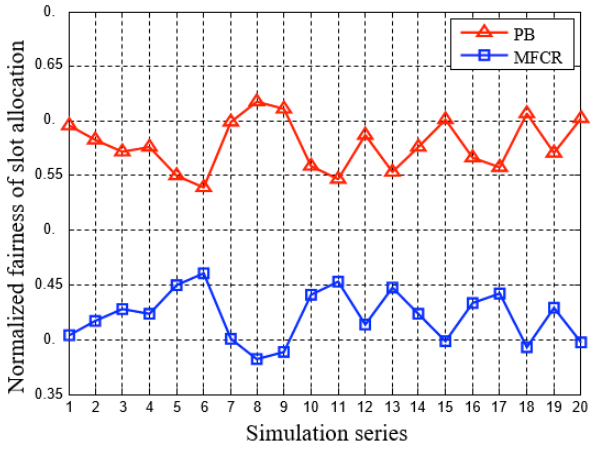

(a) Normalized fairness of slot allocation

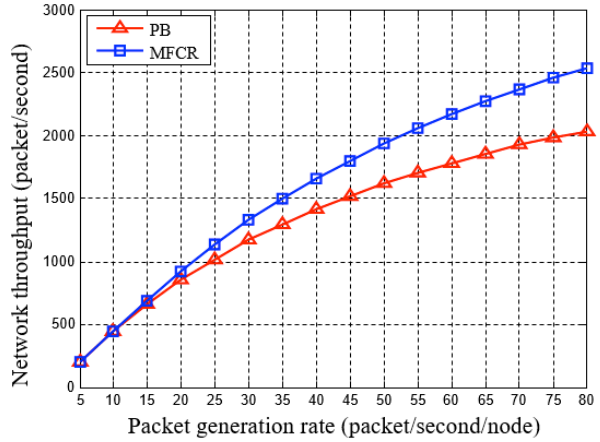

(b) Network throughput vs packet generation rate

Fig. 2 Comparison of the fairness and the network throughput.

As shown in Fig. 2(a), the variance of the slot number per frame of MFCR is less than PB. In MFCR, the node successfully reserving a slot will set the contention probability to the smallest value, and the possibility to participate in the next slot competition decreases fast. However, in PB algorithm, the node that successfully reserves a slot will participate in the next slot competition with the unchanged probability, while the competition probability of those nodes suffering continuous conflict is reduced, which will result in unfairness of slot 
allocation. Therefore, MFCR performs better than PB algorithm on the fairness of slot allocation.

\subsection{Network communication performance}

The number of nodes is 50 . The network topology is randomly generated according to the node number. The network throughput, the average access delay and the packet delivery rate are achieved respectively under conditions of different packet generation rate.

Fig. 2(b) is the network throughput comparison, the difference between the MFCR and the PB algorithms isn't obvious when the packet generation rate is low due to the light load. With the increase of the packet generation rate, because of the higher slot utilization and the fairness of slot allocation, the throughput of MFCR is higher than PB. With the increase of the packet generation rate, the throughput of these two algorithms both tend to saturated due to the limited slot resources.

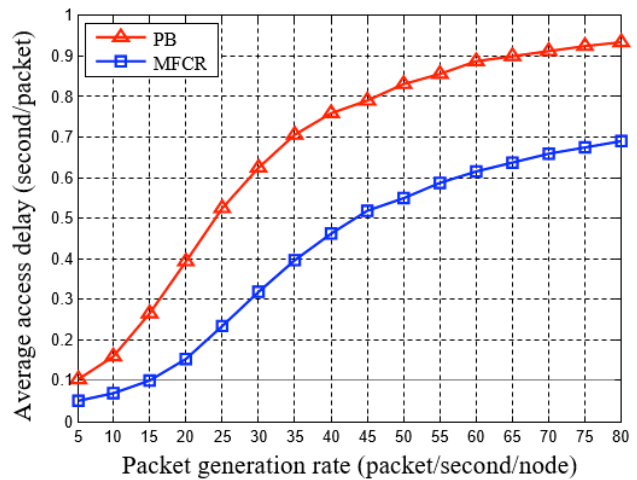

(a) Average access delay vs packet generation rate

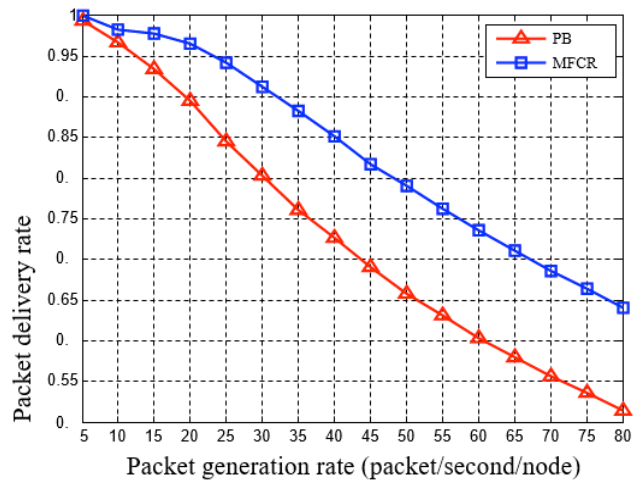

(b) Packet delivery rate vs packet generation rate

Fig. 3 Comparison of the average access delay and the packet delivery rate.

As shown in Fig. 3(a), with the increase of packet generation rate, the number of the packets that accumulated in the send buffer increase rapidly, so the average access delay in the network gradually increases. However, owing to the higher time slot utilization and the higher success reservation rate, the average access delay of MFCR performs better than PB.

Fig. 3(b) is the packet delivery rate comparison. When the packet transmission rate is lower than the packet arrival rate, the send buffer will overflow and the new arrival packets will be discarded. Hence, the packet delivery rate will decrease. Because of the higher spatial reuse capability, more packets are sent simultaneously at different node beyond two hops, the packet delivery rate of MFCR is higher than PB algorithm.

\section{Conclusion}

In this paper, we propose a multi-hop fast conflict resolution algorithm, namely MFCR for ad hoc networks. We make detailed introductions of the theoretical model, the pseudo Bayesian analysis and the algorithm design. MFCR employs a competitive probability forecasting method, which is similar to the back-off counter used in single-hop FCR, combined with the handshake process used in multi-hop pseudo Bayesian to solve the utilization and the fairness problems in distributed time slot allocation. We compared the network throughput, the packet delivery rate, the average access delay, etc. between MFCR and PB. Simulations show that MFCR has evident improvement in the network performance. 
Acknowledgement: This work was supported in part by State Grid Corporation of China 2015 Scientific Research Project under grant no. 521999150031, and the National Natural Science Foundation of China under grant no. 61271168.

\section{References}

1. C. Chrysostomou, L. Lambrinos, C. Djouvas. The need for a system design methodology to address challenges in wireless access in vehicular ad hoc networks. International Congress on Ultra Modern Telecommunications and Control Systems, pp. $1226-1231,2010$.

2. M.R. Mili, L. Musavian, K.A. Hamdi, F. Marvasti. How to Increase Energy Efficiency in Cognitive Radio Networks. IEEE Transactions on Communications, Vol. 64, no. 5, pp. 1829 - 1843, 2016.

3. Y.P. Zhang, B. Li, M. Yang, Z.J. Yan. Capacity analysis of wireless ad hoc networks with improved channel reservation. 2015 IEEE Wireless Communications and Networking Conference (WCNC), pp. 1189 - 1194, 2015.

4. Y. Kim, F. Baccelli, G. Veciana . Spatial Reuse and Fairness of Ad Hoc Networks With Channel-Aware CSMA Protocols. IEEE Transactions on Information Theory, Vol. 60, no. 7, pp. 4139 - 4157, 2014.

5. X.X. Jiang, D.H.C. Du. PTMAC: A Prediction-Based TDMA MAC Protocol for Reducing Packet Collisions in VANET. IEEE Transactions on Vehicular Technology Vol. 65, no. 11, pp. 9209 - 9223, 2016.

6. M. Sami, N.K. Noordin, M. Khabazian. A TDMA-Based Cooperative MAC Protocol for Cognitive Networks With Opportunistic Energy Harvesting. IEEE Communications Letters, Vol. 20, no. 4, pp. 808 - 811, 2016.

7. M. Hadded, P. Muhlethaler, A. Laouiti, R. Zagrouba, L.A. Saidane. TDMA-Based MAC Protocols for Vehicular Ad Hoc Networks: A Survey, Qualitative Analysis, and Open Research Issues. IEEE Communications Surveys \& Tutorials, Vol. 17, no. 4, pp. $2461-2492,2015$.

8. B. Shrestha, K.W. Choi, E. Hossain. A Dynamic Time Slot Allocation Scheme for Hybrid CSMA/TDMA MAC Protocol. IEEE Wireless Communications Letters, Vol. 2, no. 5 , pp. 535 - 538, 2013.

9. C.X. Zhu, M.S. Corson. A Five-Phase Reservation Protocol (FPRP) for Mobile Ad Hoc Networks. Wireless networks, Vol. 7, no. 4, pp. 371-384, 2001.

10. D. Arivudainambi, D. Rekha. Broadcast Scheduling Problem in TDMA Ad Hoc Networks using Immune Genetic Algorithm. International Journal of Computers Communications \& Control, Vol. 8, no. 1, pp. 18-29, 2012.

11. V. Aggarwal, N.K. Shankaranarayanan. Performance of a Random-Access Wireless Network With a Mix of Full- and Half-Duplex Stations. IEEE Communications Letters, Vol. 17, no. 11, pp. 2200 - 2203, 2013.

12. D.Y. Kim, H. Nam, H. Jin. Pseudo-Bayesian Broadcasting Algorithm for Opportunistic Splitting Scheduling Systems. IEEE Transactions on Vehicular Technology, Vo. PP, no. 99, pp. 1 - 6, 2016.

13. Y. Kwon, Y.G. Fang, H. Latchman. Design of MAC protocols with fast collision resolution for wireless local area networks. IEEE Transactions on Wireless Communications, Vol. 3, no. 3, pp. 793 - 807, 2004.

14. M.H. Chaudhary, B. Scheers. High spatial-reuse distributed slot assignment protocol for wireless ad hoc networks. 2012 Military Communications and Information Systems Conference (MCC), pp. 1 - 8, 2012. 